\title{
Regular and chaotic transport of discrete solitons in asymmetric potentials
}

\author{
J. Cuevas, ${ }^{1}$ B. Sánchez-Rey, ${ }^{1}$ and M. Salerno ${ }^{2}$ \\ ${ }^{1}$ Departamento de Física Aplicada I, E. U. P., Universidad de Sevilla, Virgen de África 7, 41011 Sevilla, Spain \\ ${ }^{2}$ Dipartimento di Fisica “E.R. Caianiello," CNISM and INFN-Gruppo Collegato di Salerno, Università di Salerno, \\ Via Ponte don Melillo, Fisciano, 84084 Salerno, Italy \\ (Received 24 March 2010; published 20 July 2010)
}

\begin{abstract}
Ratchet dynamics of topological solitons of the forced and damped discrete double sine-Gordon system are studied. Directed transport occurring both in regular and in chaotic regions of the phase space and its dependence on damping, amplitude, and frequency of the driving, asymmetry parameter, and coupling constant, has been extensively investigated. We show that the passage from ratchet phase-locked regime to chaotic ratchets occurs via a period doubling route to chaos and that, quite surprisingly, pinned states can exist inside phase locking and chaotic transport regions for intermediate values of the coupling constant. The possibility to control chaotic discrete soliton ratchets by means of both small subharmonic signals and more general periodic drivings, has also been investigated.
\end{abstract}

DOI: 10.1103/PhysRevE.82.016604

PACS number(s): 05.45.Yv, 63.20.Ry

\section{INTRODUCTION}

Ratchet dynamics in nonlinear systems are presently attracting a great deal of interest due to their relevance in several fields, including molecular motors and Josephson junctions $[1,2]$. For point particles ratchets the directed motion achieved in presence of damping and deterministic forces of zero average, is a direct consequence of the breaking of the spatiotemporal symmetries in the system [3-6]. The resulting net motion of the particle is usually phase locked to the external driving $[7,8]$ and the deterministic ratchet dynamics is quite robust to exist also in presence of noise.

Ratchet motion occurring in infinite-dimensional continuous nonlinear systems with solitonlike solutions (soliton ratchets) have been extensively investigated in the past years. This has been done both in the case of spatially asymmetric potentials and single harmonic driving $[9,10]$ and for symmetric potentials and temporarily asymmetric bihharmonic forces [6,11-13]. Experimental implementations of soliton ratchets in long Josephson junctions were also done in [14] using time-asymmetric biharmonic currents, and in $[15,16]$ using asymmetric magnetic fields or spatially asymmetric currents.

The mechanism underlying continuous soliton ratchets, also known as internal mode mechanism, is ascribed to the coupling between the soliton internal degree of freedom (internal mode) and the translational mode of the soliton [10]. This coupling is induced mainly in the presence of damping and allows an asymmetric transfer of energy from the external driving to the internal mode and to the soliton center of mass which generates the directed motion. Thus, opposite to point particle ratchets for which the action of the force is always direct on the center of mass, in soliton ratchets the force acts indirectly on the center of mass and involves the vibrational mode of the solitons in an essential manner (a direct action may exist in the overdamped limit where the phenomena becomes negligibly small [9]). This is quite natural if one thinks that the soliton is not a rigid object but it can vibrate while moving, just like a droplet of liquid does when dropping. This internal mode mechanism has been confirmed to be of general validity for continuous dissipative soliton systems $[6,10,17-20]$. The possibility of soliton ratchets in continuous Hamiltonian systems has been recently demonstrated for the case of the nonlinear Schrödinger equation with asymmetric potentials rapidly flashing in time [21].

In contrast to the continuous case, discrete soliton ratchets (DSR) in nonlinear lattices have been much less investigated. Detailed studies exist only for ratchetlike behaviors in a dissipative discrete sine-Gordon chain driven by a biharmonic force which breaks the time symmetry [22,23], and for the case of a conservative discrete nonlinear Schrödinger equation describing a one-dimensional (1D) array of coupled waveguide optical resonators [24]. In both cases the interplay between discreteness and nonlinearity introduce new features in the phenomenon such as: existence of depinning thresholds for transport, staircase like dependence of the mean drift velocity on system parameters, diffusive and even intermittent ratchetlike dynamics [22]. The possibility to enhance DSR transport by introducing phase disorder into the asymmetric biharmonic periodic driving was also considered [23].

It is interesting, however, to investigate the phenomenon of DSR in the case in which the potential is asymmetric in the field variable and the system is driven by harmonic forces of zero mean. In this case the discrete solitons have an internal degree of freedom even in absence of damping and driving, a fact which could lead to qualitative different behaviors.

The aim of this paper is twofold. From one side, we extend previous investigations on DSR to the case of asymmetric potentials. For this we consider the case of a double sineGordon chain with potential function given by

$$
V(x)=C-\frac{1}{4 \pi^{2}} \cos (2 \pi x)+\frac{\lambda}{8 \pi^{2}} \sin (4 \pi x), \quad \lambda \in[0,1],
$$

where $\lambda$ is an asymmetry parameter and $C$ is a constant that fixes the zero of the potential. Therefore the system we are going to consider belongs to the Frenkel-Kontorova family [25] and can be visualized as a chain of mechanical double 
pendula connected by a gear of ratio $1 / 2$ with nearestneighbor interactions and with the periodic on-site doublesine potential in Eq. (1) due to the gravity [26]. The dependence of the transport on the damping constant, amplitude and frequency of the driving force, coupling constant and asymmetry parameter, is investigated in detail. As a result we show that in contrast to DSR driven by asymmetric forces of zero mean [22], kink transport in the present system can be quite effective not only in correspondence of regular (phaselocked) orbits, but also on chaotic trajectories.

From the other side, we shall investigate the DSR chaotic transport in some detail both with respect to the onset of chaos and with respect to the possibility of controlling the chaotic ratchet trajectories using subharmonic signals which do not break the time symmetry in the system. To this regard, we introduce a suitable map in the space of the kink coordinates by means of which we construct bifurcation patterns. Using this approach, we show that the chaos onset occurs via period doubling and that the chaotic region can be strongly reduced in favor of periodic phase-locked orbits in presence of small subharmonic signals. The complete suppression of chaos is also investigated by means of elliptic driving functions with modulus taken as a free parameter to achieve optimal control. For the regions of parameters we have investigated, we show that the full chaos suppression can be implemented in any practical context by means of two properly chosen harmonic signals.

The paper is organized as follows. In Sec. II, we introduce the model equations and discuss the main properties of the static kink solutions, including their Peirls-Nabarro barrier and the small oscillation problems. In Sec. III, we investigate the dependence of the discrete ratchet dynamics, both phaselocked and chaotic, on the system parameters. In Sec. IV, we concentrate on the ratchet dynamics associated to chaotic orbits and on their stabilization by means of small subharmonic modulations of the harmonic driving. We study the onset of chaos by means of a suitable bifurcation map and show that chaos occurs via period doubling. Finally, in Sec. $\mathrm{V}$, we summarize the main conclusions of the paper.

\section{MODEL EQUATION}

The chain of double pendula associated to potential Eq. (1) is described in a dimensionless form by the equation [26]

$$
\ddot{x}_{n}+\alpha \dot{x}_{n}+V^{\prime}\left(x_{n}\right)+\kappa\left(2 x_{n}-x_{n+1}-x_{n-1}\right)=\gamma+F(t)+\xi_{n}(t),
$$

with $n \in[-N, N], x_{n}$ being the displacement of the nth pendulum with respect to its equilibrium position, $\gamma$ a constant bias term, $\alpha$ the damping coefficient, $\kappa$ the coupling constant which measures the discreteness of the lattice,

$$
F(t)=\frac{\varepsilon}{2 \pi} \cos (\omega t+\theta),
$$

an external symmetric in time periodic driving force of strength $\varepsilon$, frequency $\omega$ and $\xi_{n}(t)$ a stochastic term of zero average and autocorrelation function fixed as
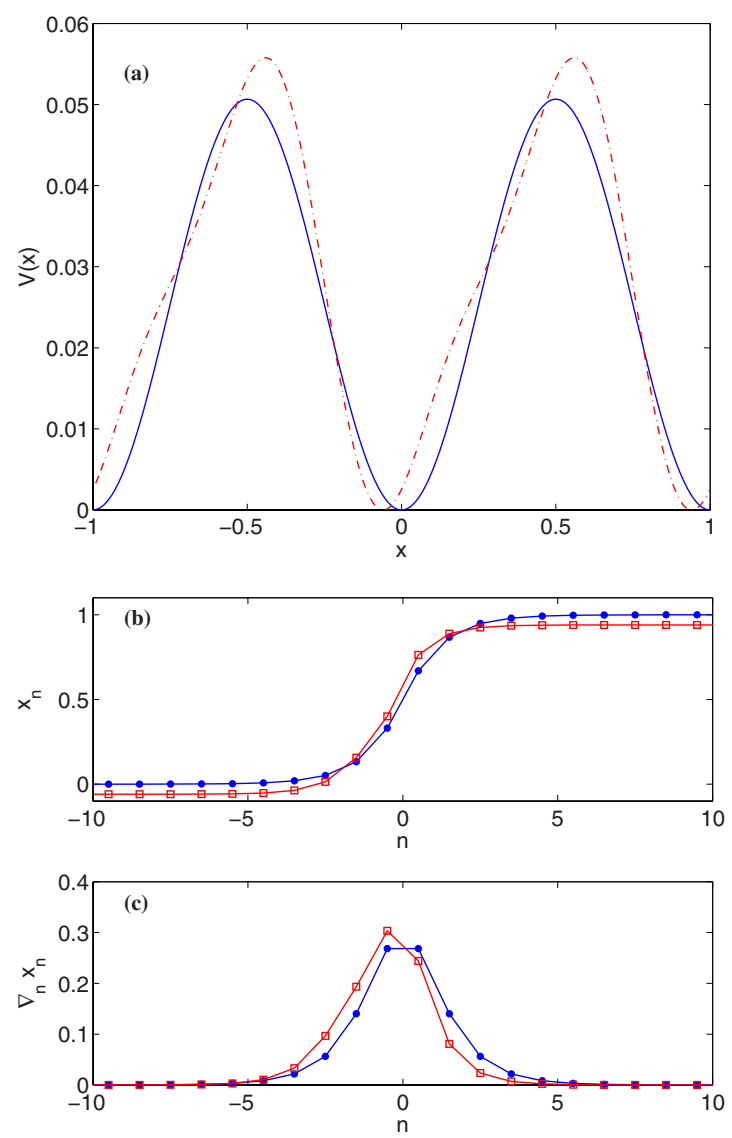

FIG. 1. (Color online) (a) Comparison between the symmetric potential for $\lambda=0$ (continuous line) and an asymmetric double sineGordon potential (dashed line) for $\lambda=0.5$. (b) Stationary discrete kink solutions of the unperturbed double sine-Gordon chain corresponding to $\lambda=0$ (circles joined by a continuous line) and $\lambda=0.5$ (squares joined by a continuous line). The discrete spatial derivatives of the kink profiles are also shown in (c) with corresponding symbols. The value of the coupling constant $\kappa$ is fixed to 1 .

$$
\left\langle\xi_{n}(t) \xi_{m}\left(t^{\prime}\right)\right\rangle=\frac{\alpha D}{\pi} \delta_{n m} \delta\left(t-t^{\prime}\right),
$$

and modeling the effect of a thermal bath in contact with the system. In the following we will investigate mainly deterministic discrete ratchets in absence of the dc bias $\gamma=0$ and in presence of the ac driving of zero average Eq. (3). We shall introduce external noise in the system only to check that the directed motion is associated to single stable attractors, a fact which permits to avoid averaging on initial conditions (e.g., initial phase $\theta$ of the driving).

In Fig. 1(a) we show the potential chain in Eq. (1) for the case $\lambda=0.5$. Notice that minima of the potential are located at $n-\delta(\lambda)$, being $\delta(\lambda)=\frac{1}{2 \pi} \sin ^{-1}\left[\frac{1-\sqrt{1+8 \lambda^{2}}}{4 \lambda}\right]$. Since a discrete kink (antikink) can be thought as a string in space extending from one absolute minimum of the potential to an adjacent one, we will assume $\lambda \in[0,1]$ to avoid the appearance of relative minima in the potential. For large coupling constant $\kappa$, approximated analytical expressions for kinks and antikinks can be obtained from the corresponding exact solutions of the continuum limit derived in [10] (notice that for large $\kappa$ 
the discrete chain is well approximated by the continuous double sine-Gordon equation), as

$$
x_{n}^{ \pm}=\delta(\lambda)+\frac{2}{\pi} \tan ^{-1}\left\{\frac{\operatorname{sgn}(\lambda) A B}{A-1-\eta \sinh \left[ \pm \frac{\Gamma_{n}}{2} \sqrt{\frac{A B}{|\lambda|}}\right]}\right\},
$$

with $A=\sqrt{1+8 \lambda^{2}}, \quad \eta=2 \lambda \sqrt{2(1+A)}, \quad B=\sqrt{2\left(4 \lambda^{2}-1+A\right)}$, and $\Gamma_{n}=\frac{n-V t}{\sqrt{1-V^{2}}}$ (the plus and minus signs refer to the kink and antikink solutions, respectively). For small values of $\kappa$ the kink solutions must be found numerically. In the panel (b) of Fig. 1 we depict two discrete kinks obtained numerically for the case of symmetric $(\lambda=0)$ and asymmetric $(\lambda=0.5)$ potentials. Notice that in the symmetric case the kink is symmetric around the center [this is clearly seen from the derivative profile shown in panel 1(c)] while in the case of asymmetric potential the kink is asymmetric and the width is a little shrunk.

\section{A. Peierls-Nabarro barrier and depinning}

A main feature of DSR with respect to continuous soliton ratchets is the presence in the discrete case of the PeirlsNabarro (P-N) barrier, which introduces a depinning threshold for kink transport. To investigate the dependence of this threshold on the system parameters we consider static kink solutions of the unperturbed system solving numerically the nonlinear system of equations

$$
V^{\prime}\left(x_{n}\right)+\kappa\left(2 x_{n}-x_{n+1}-x_{n-1}\right)=0, \quad n \in[-N, N],
$$

with the kink boundary condition $x_{N}-x_{-N}=+1$. Starting from a stable kink state we can obtain other (unstable) kink profiles whose center of mass is shifted with respect to the stable state, and then find the dependence of the kink energy on the position of its center

$$
X_{c}=\sum_{n=-N}^{n=N} n \frac{x_{n+1}-x_{n-1}}{2}
$$

In Fig. 2(a) we show the resulting P-N relief for three different values of the asymmetry parameter: $\lambda=0, \lambda=0.4$, and $\lambda=0.9$. Note that while for $\lambda=0$ the PN potential possess the inversion center symmetry $V_{P N}(X)=V_{P N}(-X)$ this is not true for the case $\lambda \neq 0$, due to the asymmetry of the potential in Eq. (1). In all cases the P-N potential is a periodic function: $V_{P N}\left(X+X_{0}\right)=V_{P N}(X)$, with period $X_{0}$ equal to 1 . The difference between the maxima and minima potential energies, $\Delta E_{P N}$, represents the activation energy necessary for putting the kink in motion. In panels (b) and (c) of Fig. 2 we have also plotted the dependence of the activation energy and of the kink width defined as [27]

$$
W_{K}=1+2 \sqrt{\sum_{n=-N}^{n=N}\left(n-X_{c}\right)^{2} \frac{\left|x_{n+1}-x_{n-1}\right|}{2}},
$$

on the asymmetry parameter $\lambda$, respectively. One can see that while the activation energy is an increasing monotonic function of $\lambda$, the kink width decreases with $\lambda$ in agreement with what observed for the kink profiles in Fig. 1. It is worth
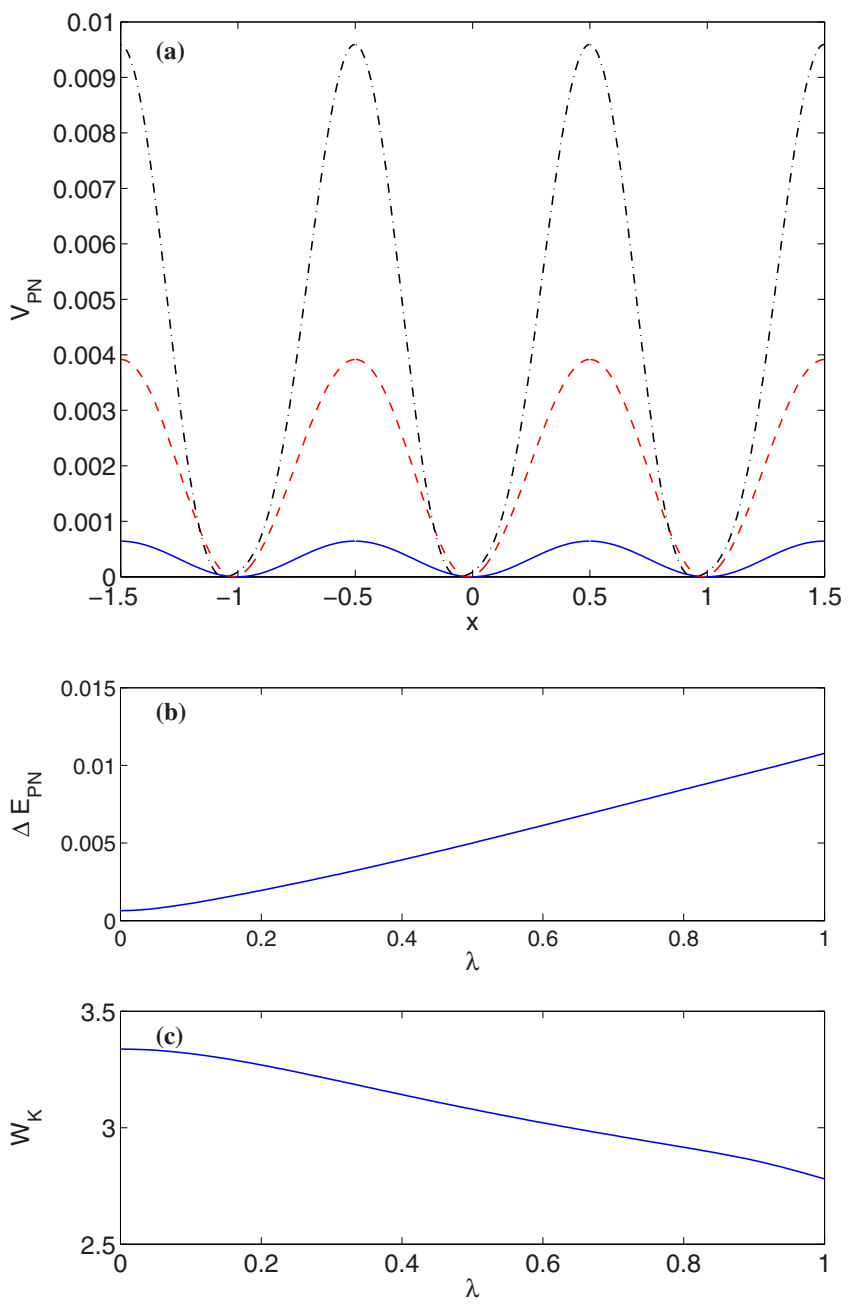

FIG. 2. (Color online) (a) Peierls-Nabarro relief for $\lambda=0$ (continuous line), $\lambda=0.4$ (dashed line) and $\lambda=0.9$ (dashed-dotted line). (b) Peierls-Nabarro energy versus lambda. (c) Dependence of the kink width on the asymmetry parameter.

noting that the above symmetry properties of the P-N potential play important role for the existence of directed kink currents in the system. In the lowest approximation, indeed, we have that the dynamics of the kink in Eq. (5) can be described in terms of an effective point-particle equation of the type [6]

$$
\frac{d^{2} X(t)}{d t^{2}}+\alpha \dot{X}(t)+V_{P N}^{\prime}(X)+\widetilde{F}(t)=0,
$$

where $X(t)$ and $\dot{X}(t)$ are collective coordinates denoting the position and the velocity of the kink, respectively, and $\widetilde{F}(t)$ is an effective driving force proportional to $F(t)$ in Eq. (3). In order to have directed transport for the kink one must break all symmetries that change sign to the kink velocity and leave the equation of motion invariant $[5,6]$ (the presence of such symmetries would imply the presence in the phase space of kink trajectories with opposite velocities, a fact which is obviously incompatible with ratchets). Since the $\mathrm{P}-\mathrm{N}$ potential in our case does not have the center inversion symmetry and since the force is symmetric in time the only 
symmetry which could link trajectories with opposite velocities is the one which is indeed an exact symmetry of Eq. (9) in absence of damping. In presence of damping, however, the time inversion symmetry is broken and so are all the symmetries of Eq. (9) which link orbits with opposite velocities. This simple point particle symmetry argument shows how important is the damping in our system to establish directed transport. It should be remarked, however, that kinks are not exactly point particles but extended objects with internal structure, which also plays an important role for directed transport, as we shall discuss in the following.

\section{B. Internal mode of discrete kinks}

As previously mentioned, discrete kinks of the double sine-Gordon system possess an oscillation of the kink profile (internal mode) even in the pure Hamiltonian limit. To investigate the existence of internal modes we have linearized the unperturbed system Eq. (6) around the static kink solution. The numerical spectrum obtained is shown in Fig. 3(a) as a function of $\lambda$. One can see that, apart the zero mode existing for all values of $\lambda$ depicted in panel (c), an internal mode emerges for $\lambda \neq 0$ below the phonon band. Its shape, plotted in panel (b), shows a spatial asymmetry induced by the asymmetry of the potential. This mode plays a crucial role in the appearance of directed motion through its coupling with the translation of the kink.

It is worth noting that the existence of that internal mode also depends on the value of the coupling constant $\kappa$. In Fig. 4 it is shown that for a fixed value of $\lambda$ the internal mode disappears for low enough coupling. In principle one can predict that no kink net motion must exist below that critical coupling. As we will see in next section this prediction is confirmed by simulations corroborating the relevance of the internal mode in the phenomenon.

\section{PHASE-LOCKED AND CHAOTIC DSR}

As mentioned before, directed kink motion is expected when symmetries of Eq. (2) that relate kink solutions with opposite velocities are broken $[5,6]$. This is the case for the double-sine Gordon potential Eq. (1) which violates the symmetry $V(x)=V(-x)$. In order to verify the existence of kink transport and to study the dependence of the phenomenon on system parameters, we have performed numerical integration of Eq. (2) by using a fourth-order Runge-Kutta method with constant step size $\Delta t=T / 200$, being $T$ the period of the external ac force. Our system is composed of 400 particles and in our simulations static kink was taken as initial condition and aperiodic boundary conditions $x_{N}-x_{-N}=1$ were also adopted.

Three main dynamical regimes are observed: (i) no net motion; (ii) phase-locked directed motion; and (iii) chaotic directed motion. Figure 5 shows two examples of both kinds of movement. In all cases we checked that the phenomenon does not depends on the initial phase $\theta$ of the driving force Eq. (3), e.g., it is independent on the choice of the initial conditions so that one can properly talk about ratchet effect.

In the phase-locked dynamics, the kink travels $k$ sites during $l$ periods of the force, and therefore its velocity is given by
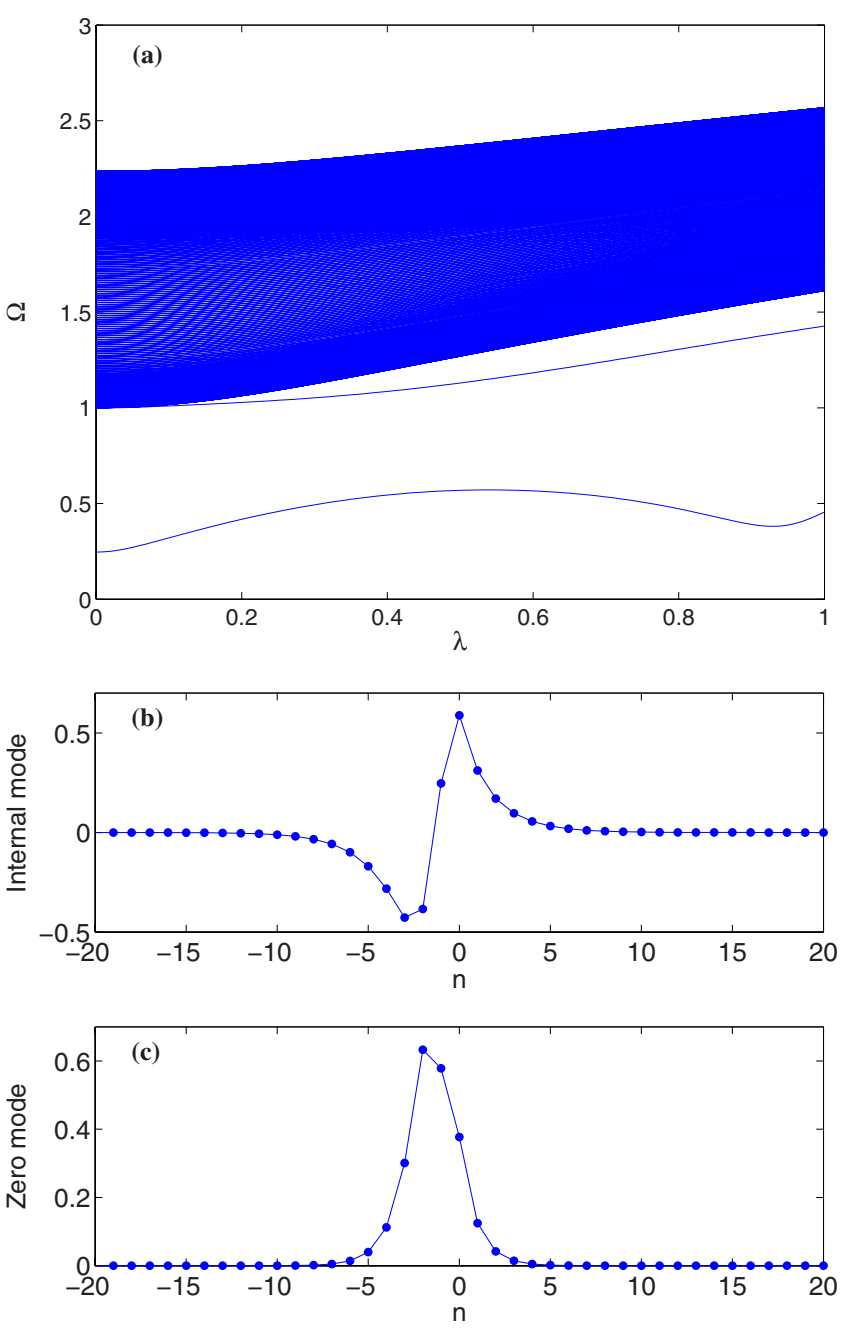

FIG. 3. (Color online) (a) Small oscillations frequency spectrum versus $\lambda$. The wide band denotes the continuum spectrum, the curve which splits off from the band corresponds to the internal mode while the bottom line refers to the translational mode (zero mode). The profiles of the internal mode and of the translational mode at $\lambda=0.46$ are shown in panels (b) and (c), respectively. The value of the coupling constant $\kappa$ is fixed to 1 .

$$
\langle v\rangle=\frac{k}{l} \frac{\omega}{2 \pi} .
$$

In this regime oscillations on the kink profile are perfectly synchronized with the motion of the kink center of mass so that the kink profile is completely reproduced after $l$ periods except for a shift in space. This suggests the existence of a coupling between internal and translational modes of the kink, similarly as for the continuous case.

In the chaotic regime the kink jumps backward and forward in an unpredictable manner and the directed motion can only be observed on a large time scale. In this regime the kink motion is of diffusive type and resembles the one of a Brownian particle. We remark that the directionality of the motion in both cases is preserved also in presence of a noise term of type Eq. (4), as one can see from Fig. 5. This is a consequence of the fact that in the phase space there is only 


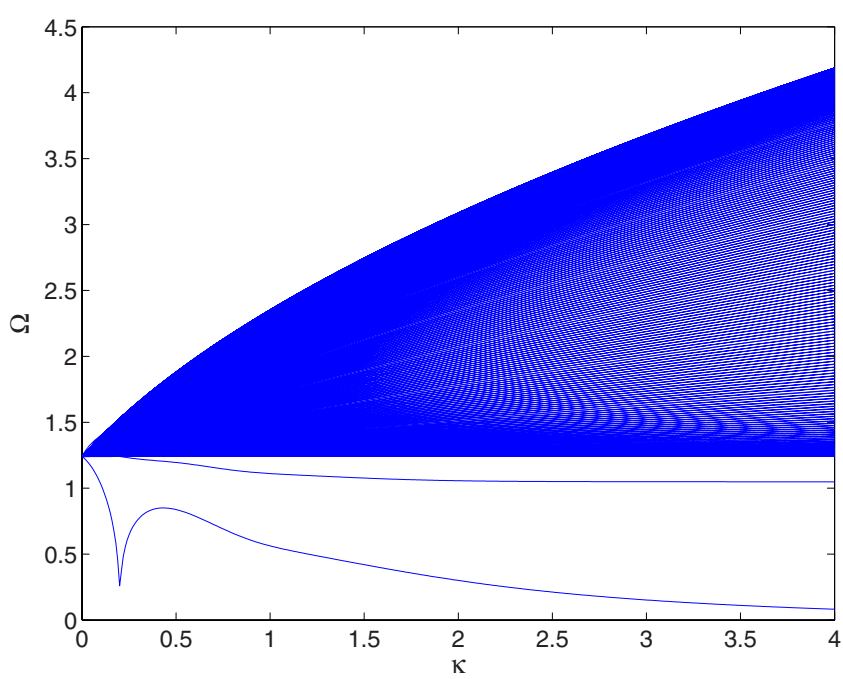

FIG. 4. (Color online) Small oscillations spectrum versus $\kappa$ for $\lambda=0.46$. Notice the disappearance of the internal mode associated with the oscillations of the kink width for $\kappa \leq 0.20$

one attractor corresponding to the net motion of the kink (in all investigated cases we have not found phenomena of hysteresis signaling the presence of more attractors for the same set of parameters). This allows to concentrate on the deterministic phenomenon and compute the mean kink velocity by averaging on time but not on the initial conditions.

Although in principle the spatial symmetry of our system is broken for any value of $\lambda \neq 0$, a distinctive feature of discrete systems is the existence of a depinning threshold above which the transport can occur and below which the kink is pinned to a lattice site. We have used to compute it a dc bias $\gamma$ instead of an ac force. The result is plotted in Fig. 6 as a function of the asymmetry parameter $\lambda$. The depinning threshold increases monotonously with $\lambda$ in accordance with the rise of the P-N barrier shown in Fig. 2. For a fixed amplitude $\varepsilon$ of the ac force the dependence of the mean kink velocity on $\lambda$ is shown in Fig. 7. It is interesting to note that even for driving amplitudes above the depinning threshold a minimal value of the asymmetry parameter is required for the directed motion to start. By further increasing $\lambda$ a rather complex scenario appears. Chaotic transport plays a dominant role. Notice that the mean drift velocity on chaotic orbits can be comparable in some cases with the one achieved in the phase-locked regime. Kink dynamics is very sensitive to parameter values and small changes of them give raise to sudden changes of the dynamical regime or can even cause a current inversion. This last finding is actually a striking result since in the continuous case the sign of $\lambda$ completely determines the sense of net motion [19]. Nevertheless, we have checked that the symmetry property $\langle v(\lambda)\rangle=-\langle v(-\lambda)\rangle$ existing in the continuous case is preserved in the case of phaselocked dynamics. The window of mobility widens of course if we increase $\varepsilon$ and more resonances appear [see Fig. 7(b)] but the scenario is qualitatively the same. In Fig. 8 we have shown the distribution of regular, chaotic and no transport regimes in the $(\varepsilon, \lambda)$ plane. We see that whereas the regular kink transport occurs mainly at small values of $\lambda$ (e.g., in the region $\lambda<0.3,0.3<\varepsilon<0.5$ ), chaotic directed motion be-
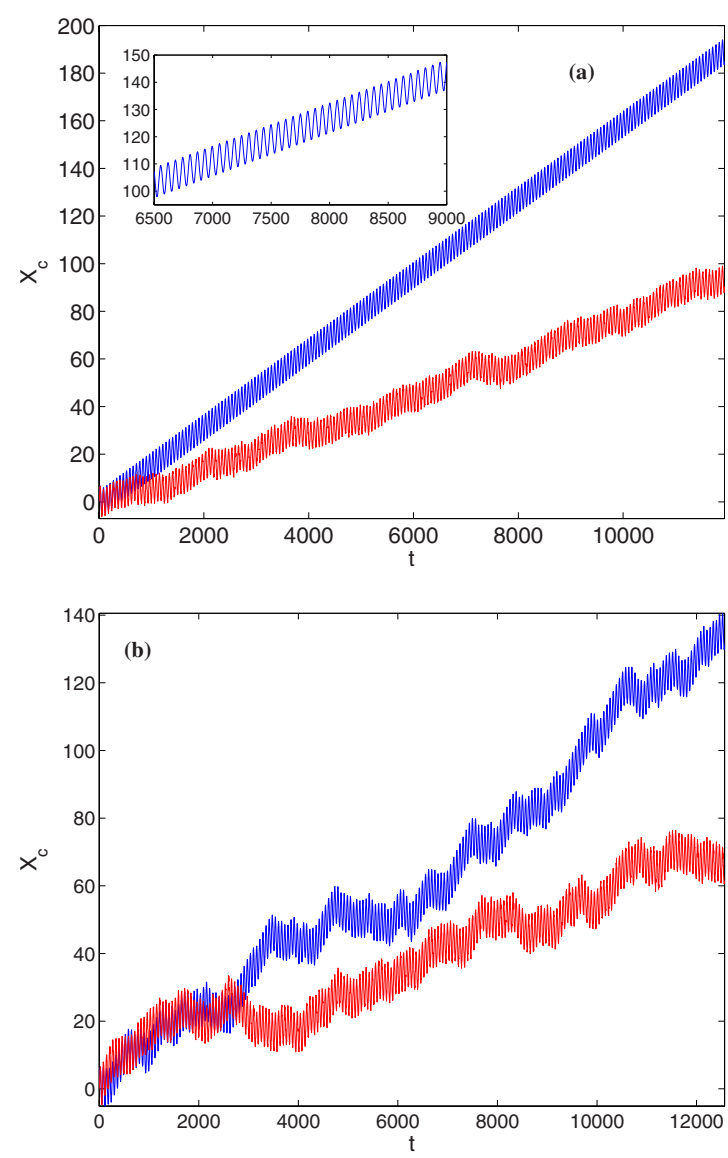

FIG. 5. (Color online) (a) the upper blue line represents phaselocked ratchet dynamics of the kink center of mass in the absence of noise for $\lambda=0.46$ and $\varepsilon=0.25$. The inset shows the perfect synchronization of the motion in a smaller time scale. When some noise $\left(D=10^{-4}\right)$ is added the direction of motion is preserved (bottom red line). (b) Chaotic ratchet dynamics for $\lambda=0.46$ and $\varepsilon=0.24$. Upper blue line corresponds to the deterministic case while bottom red line corresponds to stochastic dynamics with $D=10^{-4}$. In all cases, $\alpha$ $=0.1, \omega=0.1$, and $\kappa=1$.

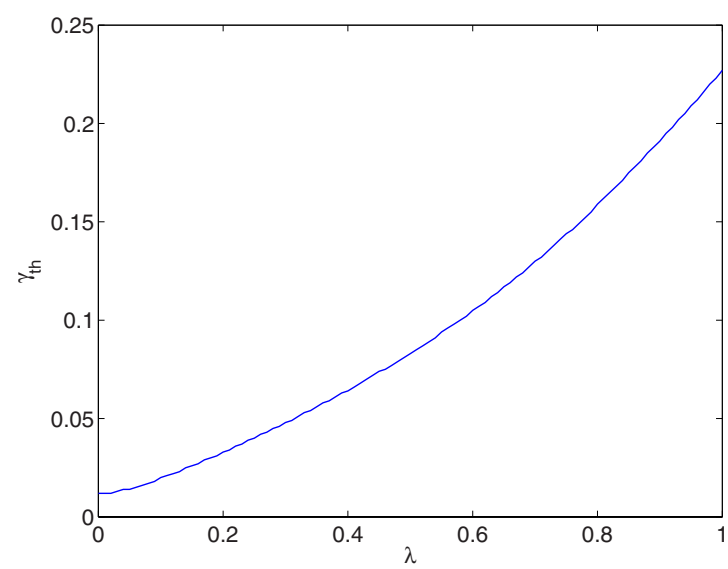

FIG. 6. (Color online) Depinning threshold for a dc bias $\gamma$ as a function of the asymmetry parameter $\lambda$. Parameters: $\alpha=0.1, \omega$ $=0.1, \varepsilon=0$, and $\kappa=1$. 

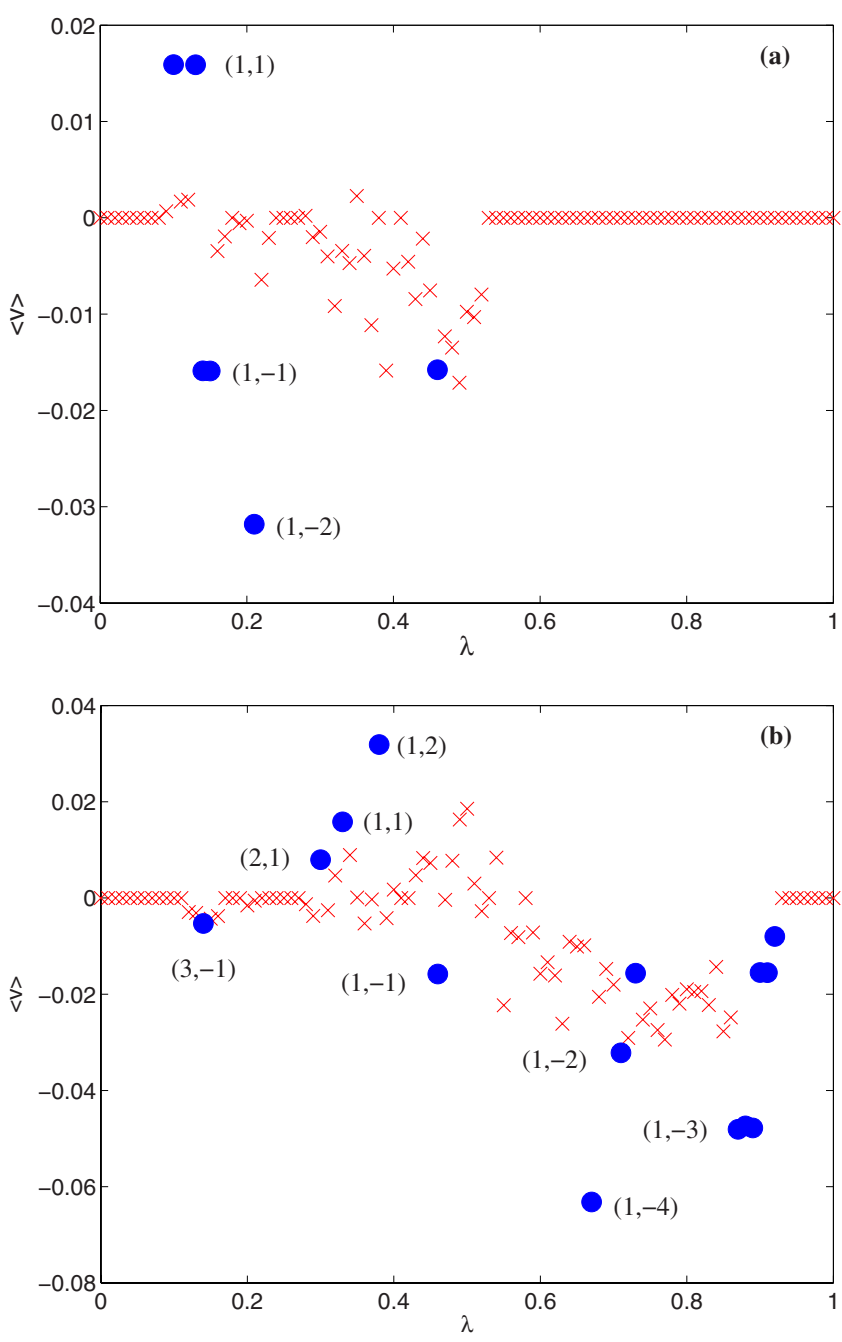

FIG. 7. (Color online) Dependence of $\langle v\rangle$ with respect to $\lambda$ for $\varepsilon=0.1$ (a) and $\varepsilon=0.2$ (b). Full circles (crosses) correspond to phaselocked (chaotic) dynamics. Numbers in brackets corresponds to the $(l, k)$ pair of Eq. (10). In both panels $\alpha=0.1, \omega=0.1$, and $\kappa=1$.

comes dominant at large values of $\lambda$. Notice also the presence of a large pinning region for small values of $\lambda$ which extends up to intermediate values of $\varepsilon$.

The influence on the phenomenon of the damping constant, $\alpha$, has also been investigated. In Fig. 9(a), we have plotted a diagram with the phase-locked attractors found on the $(\varepsilon, \alpha)$ plane for fixed $\lambda=0.46$. We have used in the numerical calculations a step of 0.01 in the damping coefficient and a thinner step of 0.005 in the amplitude of the driving force. A band structure emerges as $\alpha$ increases and, significantly, no phase-locked attractors are found when $\alpha \rightarrow 0$, showing the importance of damping in the coupling between the translational an internal modes of the kink $[19,20]$. In panel (b) of Fig. 9 we show the fraction of phase-locked attractors found for each value of damping; from it, we can observe a non-monotonous behavior of the curve and higher probability of phase locking orbits at intermediate damping.

In Fig. 10(a), we report chaotic and phase-locked attractors found by scanning the $(\lambda, \alpha)$ plane by keeping the rest of parameters fixed. Again, chaotic behavior is clearly dominant for low damping while the highest probability to find a regu-

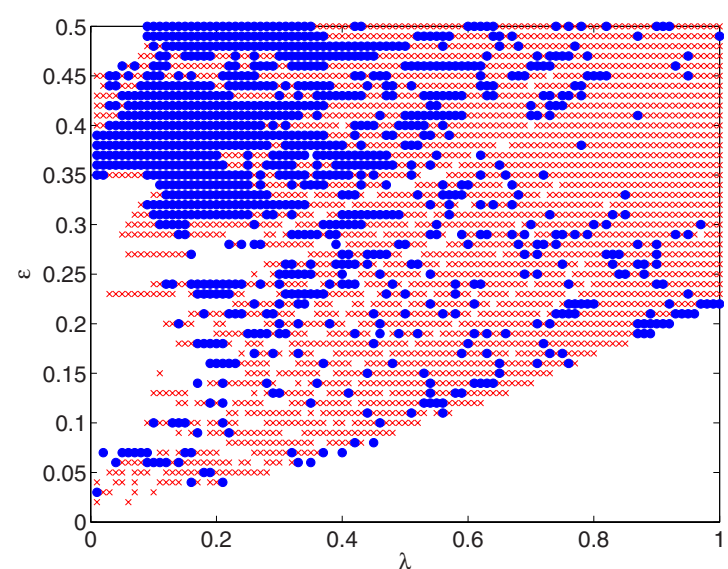

FIG. 8. (Color online) Dynamical regimes of the kink motion for different values of the asymmetry parameter $\lambda$ and the driving amplitude $\varepsilon$. Full circles (crosses) correspond to phase-locked (chaotic) dynamics. Blank spaces stand for pinned-kink states. Parameters: $\alpha=0.1, \omega=0.1$, and $\kappa=1$.

lar attractor occurs at intermediate values of the damping, as one can see in panel (b) of Fig. 10. Since in the undamped limit the kink mean velocity must go to zero (due to the
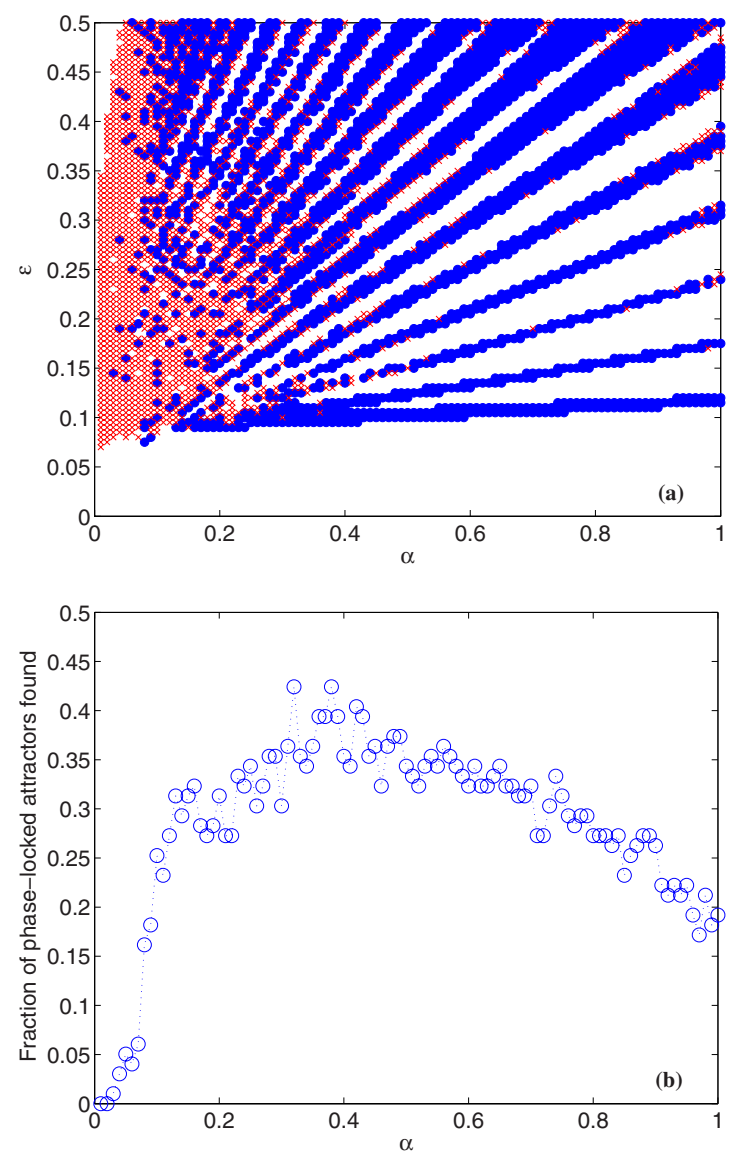

FIG. 9. (Color online) (a) Phase-locked (full circles) and chaotic (crosses) dynamical regimes of the kink ratchet motion in the $(\varepsilon, \alpha)$ plane. Blank spaces stand for pinned-kink states. Other parameters are: $\lambda=0.46, \omega=0.1$, and $\kappa=1$. (b) Fraction of phase-locked attractors found in panel (a) for each value of the damping. 

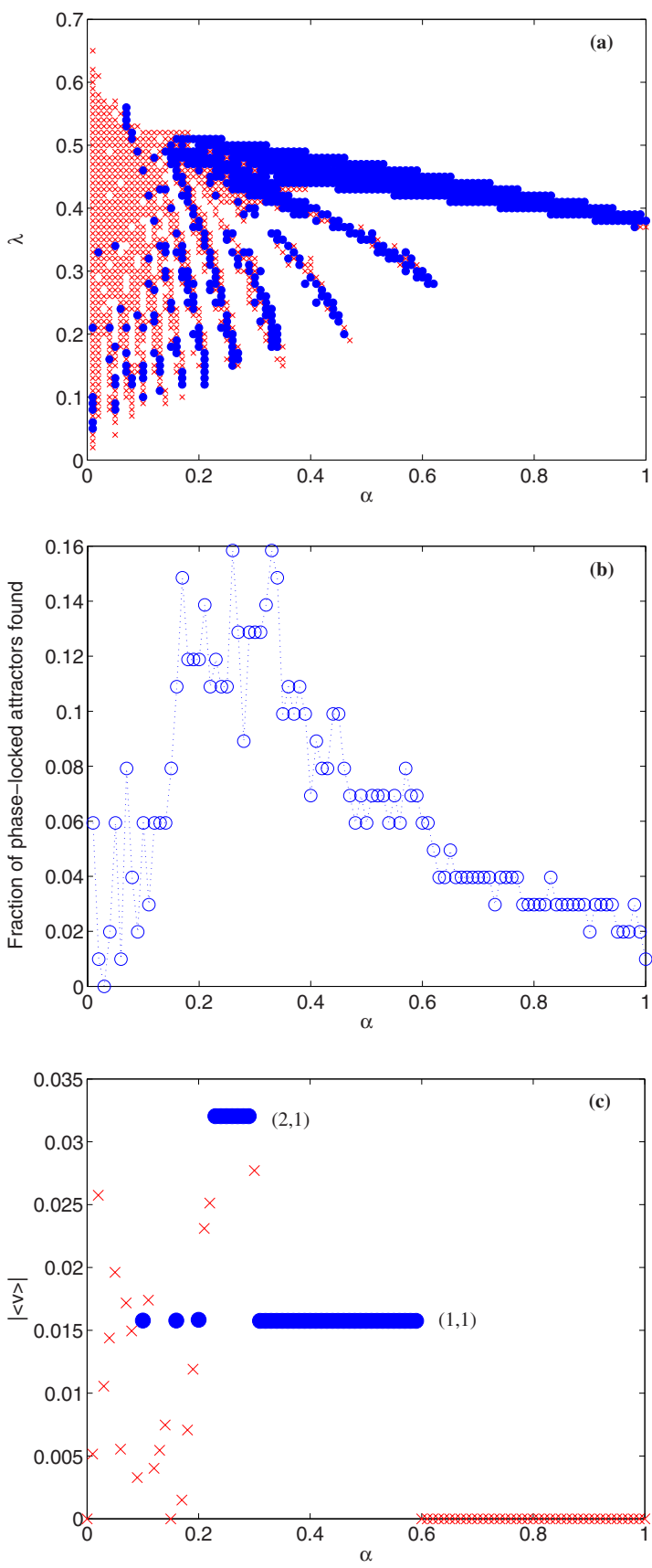

FIG. 10. (Color online) (a) Phase-locked (full circles) and chaotic (crosses) dynamical regimes of the kink ratchet motion in the $(\lambda, \alpha)$ plane. Other parameters are $\varepsilon=\omega=0.1$ and $\kappa=1$. (b) Fraction of phase-locked attractors found in panel (a) for each value of the damping. (c) Modulus of the averaged mean drift kink velocity achieved on the attractors in panel (a) for $\lambda=0.46$ as a function of the damping.

driving force which is symmetric in time), and since for large damping the internal mode mechanism is practically suppressed (this strongly reducing the drift velocity), one can expect transport not only be more probable but also more effective at intermediate values of the damping. This is indeed what is shown in panel (c) of Fig. 10 where the modulus of the averaged mean drift velocities achieved on the regular and chaotic attractors are shown as functions of the
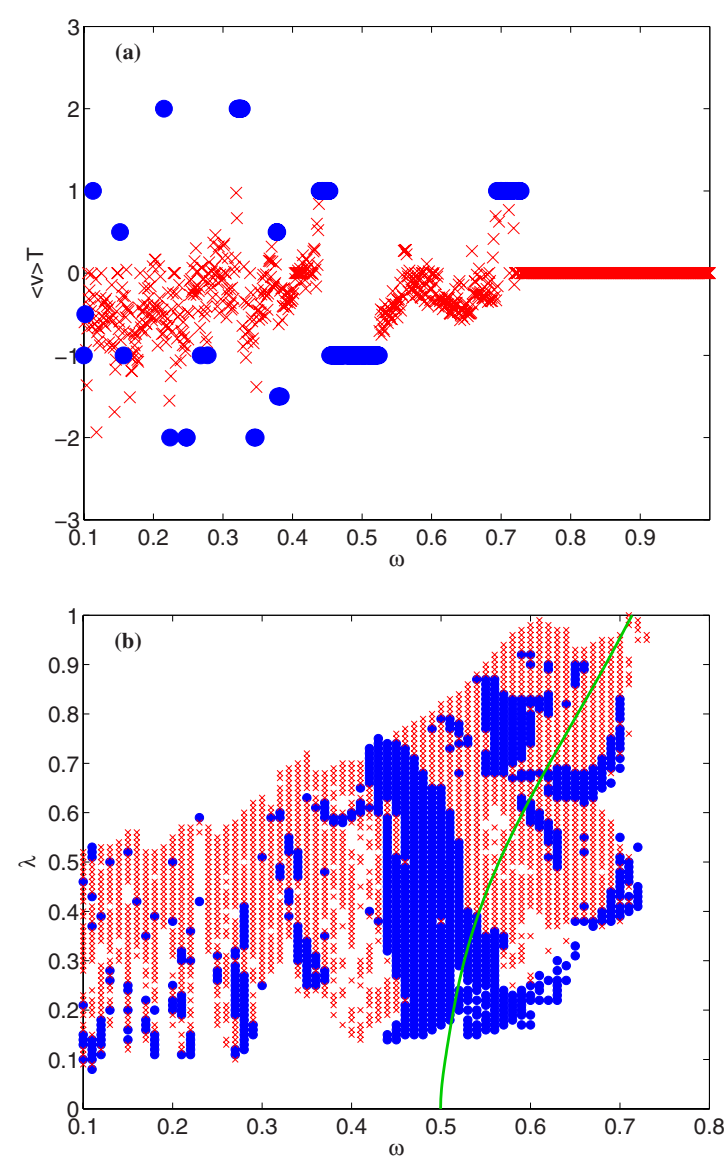

FIG. 11. (Color online) (a) Dependence of the normalized kink velocity $\langle v\rangle T$ on the driving frequency for $\varepsilon=0.1, \alpha=0.1, \lambda=0.46$, and $\kappa=1$. (b) Phase-locked (full circles) and chaotic transport (crosses) in the $(\lambda, \omega)$ plane. Blank spaces stand for pinned-kink states while the green line represents half the frequency of the unperturbed internal mode at the same value of $\lambda$.

damping. The fact that at intermediate damping the coupling between internal vibration and center of mass motion becomes optimal also correlates with similar studies performed on continuous [10,19] and discrete [22] soliton ratchets, as well as, with very recent simulations [28] of discrete kink ratchets in an asymmetric potential of polynomial type free of the Peirls-Nabarro barrier.

The dependence of the mean kink velocity on the driving frequency is characterized by the alternation of chaos with a series of phase-locking resonances. This is shown in Fig. 11(a) where we have depicted the kink mean velocity normalized to the driving frequency $\langle v\rangle T=2 \pi\langle v\rangle / \omega$ so that for phase-locked dynamics $\langle v\rangle T$ coincides with the ratio of rotation numbers $k / l$. We do not find the monotonic decay observed in the discrete sine-Gordon model with biharmonic forces [22] but, as in that case, kink transport disappears for high-enough frequencies $\omega>0.75$. Notice that the largest resonant step is achieved around $\omega=0.5$ at the rotation numbers $k=-1, l=1$, and that at low frequencies (e.g., $\omega<0.2$ ) the largest drift velocity is achieved in correspondence of chaotic trajectories. Also notice in panel (b) of Fig. 11 that around $\omega=0.5$ the phase-locked attractors cover a large region of the $(\lambda, \omega)$ plane which partially intersects the first 


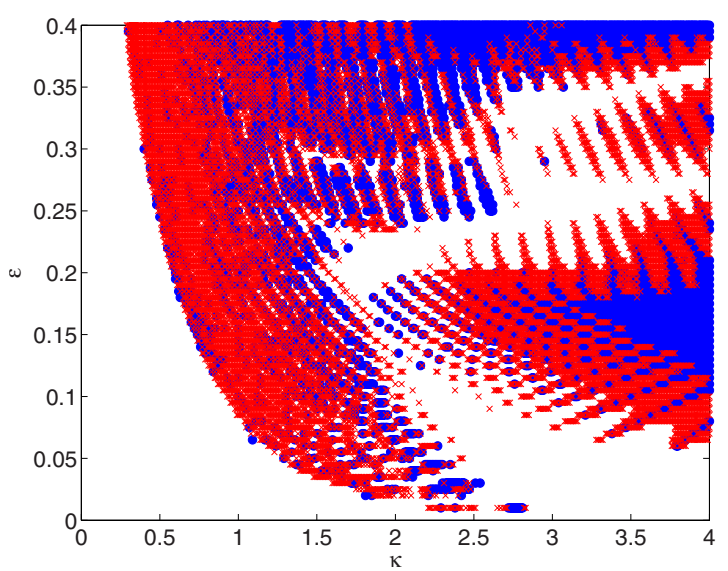

FIG. 12. (Color online) Phase-locked (full circles) and chaotic transport (crosses) in the $(\varepsilon, \kappa)$ plane. Blank spaces stand for pinned-kink states. Parameters are: $\varepsilon=0.1, \alpha=0.1, \omega=0.1$, and $\lambda$ $=0.46$.

subharmonic curve of the unperturbed internal mode frequency, this indicating a coupling between the driving frequency and the internal mode $\Omega_{I} \approx 2 \omega$.

Lastly, we have investigated the dependence of the ratchet phenomenon on the interaction constant $\kappa$. In our model this parameter measures the discreteness of the system, corresponding $\kappa \rightarrow \infty$ to the continuum limit. As expected (see Fig. 12) high discreteness prevents propagation of the kink, and the depinning amplitude of the driving decreases as $\kappa$ increases. Just above the depinning amplitude chaotic transport predominates while regular phase-locked motion becomes more important as $\kappa$ increases. Quite surprisingly, wide regions in parameter space in which the kink becomes pinned and the net motion suppressed appears for intermediate values of the interaction constant. This feature was not observed in DSR systems driven by biharmonic forces and indicates that the transport may be influenced by the local dynamics even if the driving amplitude is above the depinning threshold for a fixed value of the coupling constant $\kappa$. This unexpected transport suppression could be due to anti-resonances with the external driving or to chaos in the kink motion inside the local Peirls-Nabarro potential which prevents the escape. The precise mechanism underlying this phenomenon, however, deserves further investigations.

\section{CHAOTIC DSR CONTROL}

In contrast to continuous soliton ratchet systems which, except for the case of strongly localized inhomogeneities [29], are very robust against chaos, discrete soliton ratchets exhibit chaos particularly in the case of asymmetric potential we are dealing with. We have investigated conditions which allow to reduce the chaotic orbits in favor of the phase locked dynamics. To this regard we have added to the sinusoidal driving Eq. (3) a weak subharmonic signal $\eta \cos (\omega t / 2)$ in phase with it.

Stabilization effects induced by external weak subharmonic signals are known to be a general mechanism for suppressing chaos [30], both in point-particle systems [31] and
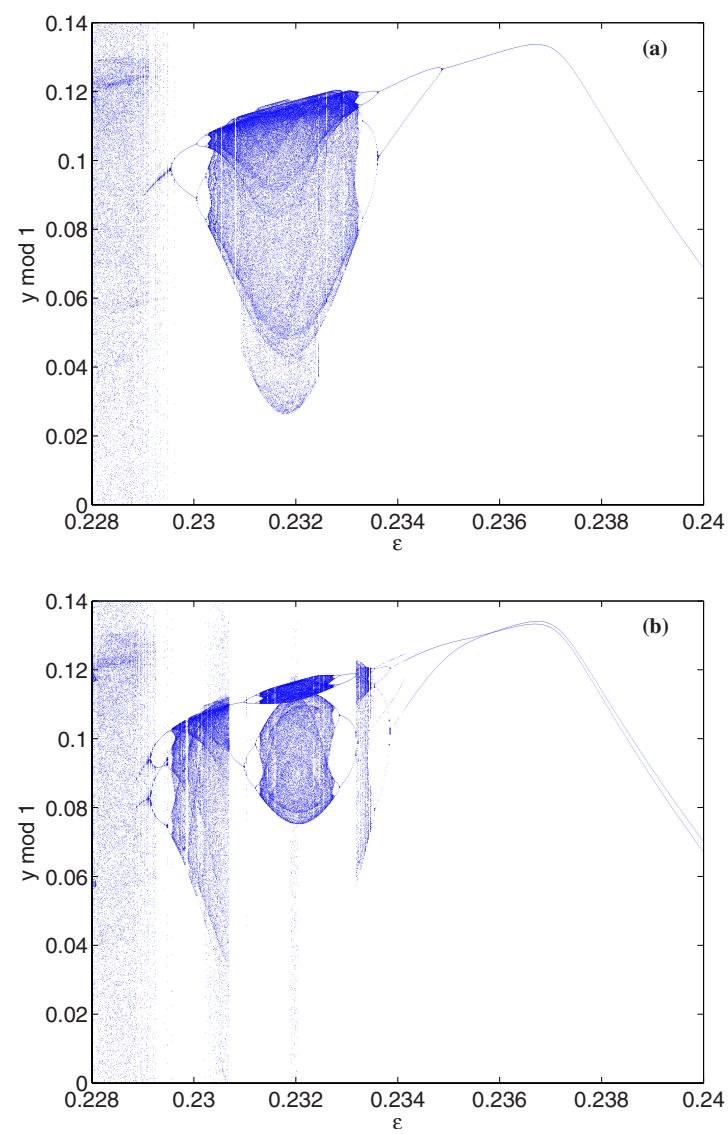

FIG. 13. (Color online) Bifurcation diagrams for $y \bmod 1$ with respect to $\varepsilon$ for $\eta=0$ (a) and $\eta=10^{-4}$ (b). Variable $y \bmod 1$ is obtained taken the position of the kink center each period of the driving after a transient. Other parameters are: $\lambda=0.4, \alpha=0.1$, and $\omega=0.2$.

in infinite degree of freedom systems described by partial differential equations [32]. The small subharmonic driving is used not to create new ratchetlike orbits but to stabilize regular orbits over larger ranges of the system parameters.

In our study we have chosen $\varepsilon$ as bifurcation parameter since, in principle, in a possible experimental implementation of the system, $\varepsilon$ would be easier to control and change than $\lambda$ for instance. For simplicity we have focused our study on an interval of $\varepsilon$ where a destabilization of phase locking orbits occurs. In Fig. 13(a), we have plotted, for $\eta=0$, a variable $y \bmod 1$ versus $\varepsilon$, where $y$ is defined as the stroboscopic map of $X_{c}$ with period $T=2 \pi / \omega$ (i.e., $X_{c}$ taken each period $T$ after a transient). One can observe phase-locked orbits represented by constant values of $y$ mod 1 for a given $\varepsilon$. Transition to chaos occurs via a cascade of period doubling bifurcations. In this graph, for the sake of continuity of the variable $y$, we have used as initial condition for each step the solution obtained at the previous one. Another stroboscopic map for the same interval and $\eta=10^{-4}$ is shown in panel (b) of Fig. 13. We see that in this case the subharmonic signal, although significantly reduces the dispersion in $y$ in the chaotic region, it does not give the full suppression of the chaotic region. This suggests that other harmonic signals may also be involved in the full chaos suppression. 

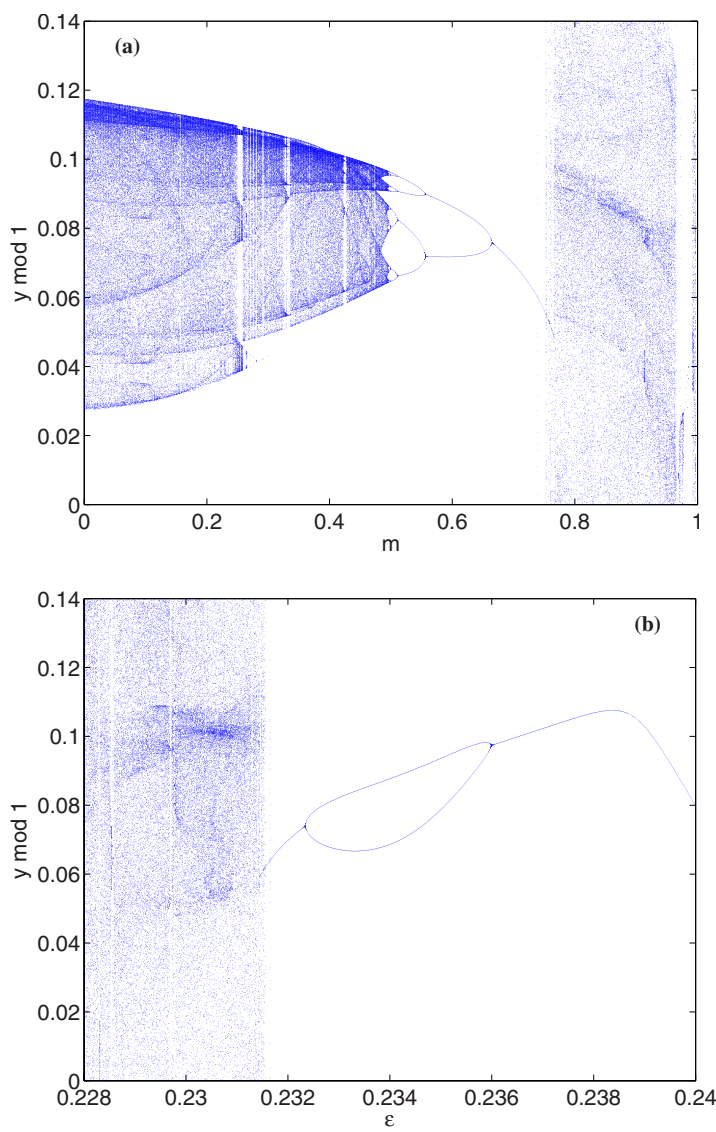

FIG. 14. (Color online) (a) bifurcation diagram for $y \bmod 1$ with respect to parameter $m$ of the elliptic function Eq. (11) and $\varepsilon$ $=0.232$. The amplitude $\varepsilon^{\prime}$ has been adjusted according to Eq. (13). (b) bifurcation diagram as a function of $\varepsilon$ for $m=0.7$. In both panels parameters are the same as in Fig. 13.

To find the optimal shape of the small signal for chaos suppression, it is convenient to use a more general driving in the form of a Jacobi elliptic function

$$
F(t)=\varepsilon^{\prime} \operatorname{cn}\left(\frac{2 K}{\pi} \omega t, m\right),
$$

where $\varepsilon^{\prime}$ is the amplitude of the driving, $0 \leq m \leq 1$ is the elliptic modulus and $K$ is the complete elliptic integral of the first kind $K(m)=\int_{0}^{\pi / 2}\left(1-m \sin ^{2} \theta\right)^{-1 / 2} d \theta$ [33]. This driving allows us to change the periodicity of the signal continuously by means of the modulus $m$ used as a free parameter to optimize the shape. An elliptic driving, apart from being periodic, satisfying the symmetry $F(t)=-F(t+T / 2)$ and reducing to a sinusoidal driving Eq. (3) in the limit $m=0$, has the advantage of being expressed in terms of sinusoidal functions as

$$
F(t)=\frac{2 \pi}{m^{1 / 2} K} \sum_{n=0}^{\infty} \frac{q^{n+1 / 2}}{1+q^{2 n+1}} \cos [(2 n+1) \omega t],
$$

with the coefficient of the series depending on the Nome $q$ $=\mathrm{e}^{-\pi K(1-m) / K(m)}$ and with only odd multiples of the fundamental frequency entering the sum. Similar drivings were also used to suppress chaotic dynamics of Bose-Einstein

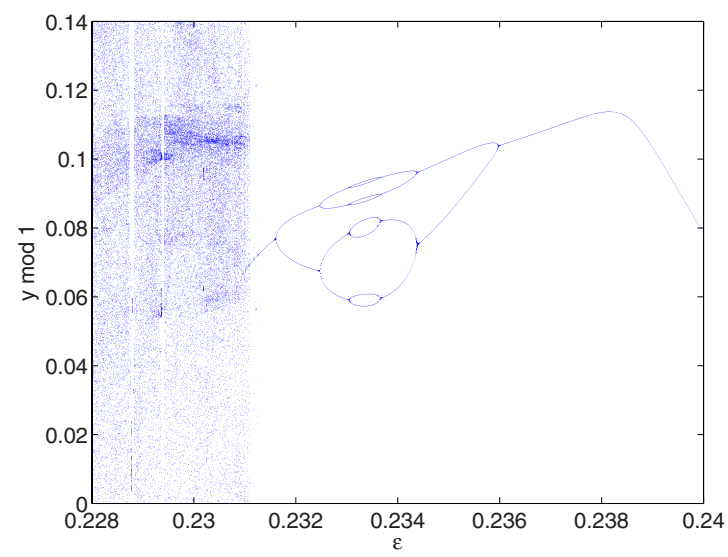

FIG. 15. (Color online) Bifurcation diagram as a function of $\varepsilon$ obtained for $m=0.7$ when the elliptic function is replaced with the first two terms of its Fourier expansion. Parameters are the same as in Fig. 13.

condensates loaded into a moving optical lattice [34].

The above driving was used to stabilize, for example, the chaotic region of our original system around $\varepsilon=0.232$ in Fig. 13(a). By replacing the sinusoidal subharmonic driving with the elliptic one Eq. (11) and starting from $m=0$, we have increased $m$ looking for a regularization route to a phaselocking dynamics. In order to properly compare with the sinusoidal case $(m=0)$ we have adjusted, while increasing $m$, the amplitude $\varepsilon^{\prime}$ so as to keep the first Fourier coefficient of the expansion Eq. (12) to be $\varepsilon$, i.e., we performed the regularization by following the path

$$
\varepsilon^{\prime}(m)=\left\{\begin{array}{ll}
\varepsilon & \text { if } m=0 \\
\varepsilon \frac{m^{1 / 2} K(1+q)}{2 \pi q^{1 / 2}} & \text { if } 0<m<1
\end{array} .\right.
$$

The results are depicted in Fig. 14(a). We see that as $m$ is increased a progressive regularization occurs. The system undergoes an inverse period doubling sequence of bifurcations until it reaches a periodic orbit around $m=0.7$. We have chosen this value to compute now a new stroboscopic map with $\varepsilon$ as bifurcation parameter which it is shown in panel (b) of Fig. 14. Comparison with Fig. 13(a) reveals the complete regularization of the system for $\varepsilon \gtrsim 0.2316$.

An elliptic driving in practical applications could be difficult to implement. We observe, however, that the main contributions in the series expansion Eq. (12) come from the first and third harmonics. Thus, for instance, for $m=0.7$ the coefficients of the third and fifth harmonics are $0.0802 \varepsilon^{\prime}$ and $0.00603 \varepsilon^{\prime}$, respectively, being $\varepsilon^{\prime}$ the amplitude of the first harmonic. In Fig. 15, we have depicted the resulting stroboscopic map when the elliptic function Eq. (11) is substituted for the first two terms of its Fourier expansion. Note that the addition of a third harmonic to the fundamental frequency is indeed enough to suppress chaos. Therefore a reasonable option in a possible experimental set-up to control chaos would be to add to the ac driving a third harmonic conveniently adjusted. 


\section{CONCLUSIONS}

We have studied the ratchet phenomena induced by asymmetric potentials on discrete topological solitons (kinks and antikinks) of the discrete damped double sine-Gordon equation in the presence of harmonic driving. The dependence of the mean velocity of the kink on the asymmetry parameter, damping, amplitude of the driving, frequency of the driving and on the coupling constant, has been extensively investigated. We have shown that in contrast with DSR driven by asymmetric forces of zero mean [22], kink transport can be quite effective not only on regular (phase-locking) orbits, as it is usual for soliton ratchets [10], but also on chaotic trajectories. In particular, we have shown that although the mean kink velocity in the chaotic region is typically smaller than the one achieved on phase-locked orbits, there are parameter values for which the chaotic transport becomes more efficient than the one in the phase locking regime [this case is observed, for example, in Fig. 11(a) for small values of the driving frequencies, e.g., for $\omega<0.2]$.

Our investigation of the dependence of the kink transport on the damping constant shows that a phase locking regime and therefore an efficient transport is more probable at intermediate values of damping. This fact correlates with previous investigations and explanations in terms of the internal mode mechanism.

The mechanism of the kink transport also depends strongly on the coupling constant. We find that for large values of the coupling constant the kink transport is dominated by the regular phase locked motion while the diffusive chaotic transport becomes important for small values of $\kappa$. Quite surprisingly, we have also found that for intermediate values of $\kappa$ a wide region of pinned kink states can exist.
The ratchet dynamics in the chaotic regime has been investigated in terms of a reduced map for the center of the kink which allows us to show that the onset of chaos occurs via period doubling bifurcations. The possibility to control chaotic ratchet dynamics has also been investigated by means of an additional sub-harmonic driving which does not break the time symmetry of the system. In particular, we have shown that the chaotic region can be strongly reduced in favor of periodic phase-locked orbits even for very small strengths of the subharmonic signal in comparison with the fundamental driving. To fully suppress chaos we have used a more general driving in the form of an elliptic function with the modulus taken as a free parameter to modulate the signal and to find the optimal regularization route for the system dynamics. For the region of parameters investigated, we have found that in any practical context this would lead to phase-locking ratchet transport and to chaos suppression simply by adding a third harmonic conveniently adjusted to the fundamental driving. Finally, we remark that the features of DSR investigated in this paper may be of general validity also for other systems undergoing structural changes which induce an asymmetry in the potential energy.

\section{ACKNOWLEDGMENTS}

We acknowledge F. Palmero for helpful comments. J.C. and B.S.-R. acknowledge financial support from the MICINN (Project No. FIS2008-04848). B.S.-R. also acknowledges financial support from the MICINN (Project No. FIS2008-02873). M.S. acknowledges partial support from a MIUR initiative about research projects of national relevance (Grant No. PRIN-2008).
[1] P. Hänggi and R. Bartussek, in Nonlinear Physics of Complex Systems-Current Status and Future Trends, Lecture Notes in Physics Vol. 476, J. Parisi, S. C. Müller, and W. Zimmermann (Springer, Berlin, 1996), p. 294; F. Jülicher, A. Ajdari, and J. Prost, Rev. Mod. Phys. 69, 1269 (1997).

[2] P. Reimann, Phys. Rep. 361, 57 (2002).

[3] S. Flach, O. Yevtushenko, and Y. Zolotaryuk, Phys. Rev. Lett. 84, 2358 (2000).

[4] O. Yevtushenko, S. Flach, Y. Zolotaryuk, and A. A. Ovchinnikov, EPL 54, 141 (2001).

[5] S. Denisov, S. Flach, A. A. Ovchinnikov, O. Yevtushenko, and Y. Zolotaryuk, Phys. Rev. E 66, 041104 (2002).

[6] M. Salerno and Y. Zolotaryuk, Phys. Rev. E 65, 056603 (2002).

[7] J. L. Mateos, Phys. Rev. Lett. 84, 258 (2000).

[8] M. Barbi and M. Salerno, Phys. Rev. E 62, 1988 (2000).

[9] F. Marchesoni, Phys. Rev. Lett. 77, 2364 (1996).

[10] M. Salerno and N. R. Quintero, Phys. Rev. E 65, 025602(R) (2002).

[11] S. Flach, Y. Zolotaryuk, A. E. Miroshnichenko, and M. V. Fistul, Phys. Rev. Lett. 88, 184101 (2002).

[12] Y. Zolotaryuk, M. Salerno, and P. L. Christiansen, Int. J. Mod. Phys. B 17, 4428 (2003).
[13] E. Goldobin, A. Sterck, and D. Koelle, Phys. Rev. E 63, 031111 (2001).

[14] A. V. Ustinov, C. Coqui, A. Kemp, Y. Zolotaryuk, and M. Salerno, Phys. Rev. Lett. 93, 087001 (2004).

[15] G. Carapella and G. Costabile, Phys. Rev. Lett. 87, 077002 (2001).

[16] M. Beck, E. Goldobin, M. Neuhaus, M. Siegel, R. Kleiner, and D. Koelle, Phys. Rev. Lett. 95, 090603 (2005).

[17] L. Morales-Molina, N. R. Quintero, F. G. Mertens, and A. Sanchez, Phys. Rev. Lett. 91, 234102 (2003).

[18] C. R. Willis and M. Farzaneh, Phys. Rev. E 69, 056612 (2004).

[19] N. R. Quintero, B. Sanchez-Rey, and M. Salerno, Phys. Rev. E 72, 016610 (2005).

[20] L. Morales-Molina, N. R. Quintero, A. Sanchez, and F. G. Mertens, Chaos 16, 013117 (2006).

[21] D. Poletti, T. J. Alexander, E. A. Ostrovskaya, B. Li, and Y. S. Kivshar, Phys. Rev. Lett. 101, 150403 (2008).

[22] Y. Zolotaryuk and M. Salerno, Phys. Rev. E 73, 066621 (2006).

[23] P. J. Martínez and R. Chacón, Phys. Rev. Lett. 100, 144101 (2008).

[24] A. Gorbach, S. Denisov and S. Flach, Opt. Lett. 31, 1702 
(2006).

[25] O. M. Braun and Yu. S. Kivshar, Phys. Rep. 306, 1 (1998).

[26] M. Salerno, Physica D 17, 227 (1985).

[27] A. V. Savin, G. P. Tsironis, and A. V. Zolotaryuk, Phys. Rev. E 56, 2457 (1997).

[28] Y. V. Bebikhov, S. V. Dmitriev, S. V. Suchkov, and A. Khare, Phys. Lett. A 374, 1477 (2010).

[29] P. Müller, F. G. Mertens, and A. R. Bishop, Phys. Rev. E 79, 016207 (2009).

[30] Y. Braiman and I. Goldhirsch, Phys. Rev. Lett. 66, 2545
(1991).

[31] M. Barbi and M. Salerno, Phys. Rev. E 63, 066212 (2001).

[32] M. Salerno and M. R. Samuelsen, Phys. Lett. A 190, 177 (1994); G. Filatrella, G. Rotoli, and M. Salerno, ibid. 178, 81 (1993).

[33] Handbook of Mathematical Functions with Formulas, Graphs and Mathematical Tables, edited by M. Abramowitz and I. Stegun (Dover, New York, 1972).

[34] R. Chacón, D. Bote, and R. Carretero-González, Phys. Rev. E 78, 036215 (2008). 